\title{
Estrogen-decreased hsa_circ_0001649 promotes stromal cell invasion in endometriosis
}

\author{
Qi Li ${ }^{1}, \mathrm{Na} \mathrm{Li}^{1}$, Hengwei Liu², Yu Du³, Haitang He ${ }^{1}$, Ling Zhang ${ }^{1}$ and Yi Liu ${ }^{1}$ \\ ${ }^{1}$ Department of Obstetrics and Gynecology, Union Hospital, Tongji Medical College, Huazhong University of \\ Science and Technology, Wuhan, China, ${ }^{2}$ Department of Obstetrics and Gynecology, Zhongnan Hospital of Wuhan \\ University, Wuhan University, Wuhan, China and ${ }^{3}$ Department of Obstetrics and Gynecology, Tongji Hospital, \\ Tongji Medical College, Huazhong University of Science and Technology, Wuhan, China
}

Correspondence should be addressed to L Zhang or Y Liu; Email: zhanglingxh@hust.edu.cn or liqun1994@hust.edu.cn

\begin{abstract}
Endometriosis (EMs) is an estrogen $\left(E_{2}\right)$-dependent inflammatory disorder. Although $E M s$ is considered a benign disease, it presents with malignant characteristics, such as migration and invasion. An increasing number of studies have shown that aberrantly expressed circular RNAs (circRNAs) play an essential role in disease development and progression. However, the mechanisms by which circRNAs exert their pathological effects in EMs remain unclear. Hsa_circ_0001649, a novel cancer-associated circRNA, has been previously reported to be downregulated in several cancer types and related to cell migration and invasion. In the present study, real-time PCR (qRT-PCR) was carried out to measure hsa_circ_0001649 levels in human tissues, human primary endometrial stromal cells (ESCs) and a human endometrial stromal cell line (ThESCs). Matrix metalloproteinase 9 (MMP9) levels in ESCs and ThESCs were assessed by qRT-PCR and Western blotting, and the migration and invasion capacities of ThESCs were evaluated by transwell assay. As a result, hsa_circ_0001649 expression was significantly decreased in ectopic and eutopic endometrial samples compared with that in normal endometrial samples. $E_{2}$ decreased hsa_circ_0001649 expression but increased MMP9 expression in ESCs and ThESCs. Furthermore, ThESCs were more invasive under $\mathrm{E}_{2}$ stimulation. However, these effects disappeared when ICI or hsa_circ_0001649 transfection was used. Collectively, our findings reveal that decreased hsa_circ_0001649 expression plays a role in $E_{2}$-increased MMP9 expression through $E_{2}$ receptors (ERs), which have critical functions in EMs.

Reproduction (2020) $\mathbf{1 6 0} 511-519$
\end{abstract}

\section{Introduction}

Endometriosis (EMs), which is histologically defined by the presence of endometrial tissue outside the endometrial cavity, is an estrogen $\left(E_{2}\right)$-dependent inflammatory disorder associated with variable phenotypic and symptomatic presentations (Yilmaz \& Bulun 2019). EMs is estimated to occur in at least $10 \%$ of reproductive-aged women, $40 \%$ of infertile women and $90 \%$ of women with pelvic pain (Kodaman 2015). Despite extensive research and various theories, the pathogenesis of EMs remains controversial (Harada et al. 2001). Moreover, EMs is an enigma due to delayed diagnosis and ineffective treatment (Kvaskoff et al. 2015). Therefore, additional basic research is needed to enhance our understanding of the etiology of EMs and identify new diagnosis and treatment options.

An increasing number of studies have shown that noncoding RNAs (ncRNAs) are functionally important for normal development and physiology as well as pathology (Esteller 2011). NcRNAs, including miRNA, long ncRNAs (IncRNAs) and circular RNAs (circRNAs)
(Anastasiadou et al. 2018), are functional RNAs transcribed from DNA but are mostly not translated into proteins (Cech \& Steitz 2014). Recently, several studies have focused on the roles of miRNAs and IncRNAs in Ems (Ferlita et al. 2018, Panir et al. 2018). We also previously found that the IncRNA MALAT1 mediates hypoxiainduced pro-survival autophagy in endometrial stromal cells (ESCs) in Ems (Liu et al. 2019) and that MALAT1 acts as a miRNA sponge for miR200, which is involved in the $\mathrm{E}_{2}$-induced epithelial-mesenchymal transition (EMT) in Ems (Du et al. 2019). However, few studies have focused on the newly discovered circRNAs in EMs. In contrast to linear RNAs, circRNAs are characterized by covalently linked terminals, high stability in the circulation, and abundant expression, rendering them ideal biomarkers for diagnostic, prognostic, and therapeutic response predictions (Qu et al. 2015). Moreover, circRNAs play a key regulatory role in gene expression at the transcriptional and posttranscriptional levels (Greene et al. 2017). Although the mechanism of action of circRNAs is unclear, several circRNAs have been reported to act as 'miRNAs sponges' to regulate miRNA 
activity (Hansen et al. 2013), to interact with RNAbinding proteins (RBPs) (Zang et al. 2020), or to bind to RNA-pol II to regulate transcription (Li et al. 2015) and even translation into specific proteins in some particular cases (Pamudurti et al. 2017). Regardless, research to date on the mechanism of circRNA in EMs is still in its infancy. Of the few studies that have investigated EMs and circRNAs, most have focused only on differences in circRNA expression in EMs and have not examined how differentially expressed circRNAs function in EMs (Shen et al. 2018, Xu et al. 2018a,b, Zhang et al. 2018, 2019, Wang et al. 2019). Thus, more effort is required to determine the mechanisms by which circRNAs exert their pathological effects on EMs.

Although EMs is considered a benign disease, it has biological behaviors similar to malignant tumors, such as invasion, distant metastasis, and recurrence. Hsa circ_0001649, a transcriptional product of the tumor suppressor gene SNF2 histone linker PHDRINGhelicase (SHPRH) (Wang et al. 2018), is a novel cancerassociated circRNA that exhibits decreased expression in several cancer types, including cholangiocarcinoma, hepatocellular carcinoma and colorectal cancer (Xing et al. 2018). Qin et al. (2016) confirmed that hsa_ circ_0001649 knockdown in vitro increases expression of matrix metalloproteinases (MMPs, including MMP9, MMP10 and MMP13) in human hepatoma cells and promotes tumor invasion and metastasis. MMP9 is an important member of the MMPs family involved in cell invasion and metastasis (van Kempen \& Coussens 2002). Moreover, MMP9 is highly expressed in endometriosis lesions (Pino et al. 2009), and in our previous research, we found that MMP9 promotion through the $\mathrm{Wnt} / \beta$ catenin pathway under $E_{2}$ regulation may contribute to the pathophysiology of Ems (Zhang et al. 2016). However, whether hsa_circ_0001649 is involved in $\mathrm{E}_{2}$ mediated MMP9 upregulation in EMs remains unknown.

In the present study, we aimed to determine whether hsa_circ_0001649 expression levels differ among eutopic, ectopic, and normal endometrial samples and whether hsa_circ_0001649 is involved in $\mathrm{E}_{2}$-mediated MMP9 upregulation in EMs. Our study provides novel insight into the circRNA-related pathogenesis of EMs.

\section{Materials and methods}

\section{Tissue collection}

Paired eutopic $(n=40)$ and ectopic $(n=40)$ endometrial samples were collected from patients with ovarian EMs undergoing laparoscopy. These patients were 21-37 years of age and classified as having endometriosis at stages I-IV according to the American Society for Reproductive Medicine (ASRM) criteria (American Society for Reproductive Medicine 1997). Normal endometrial samples $(n=101)$ were collected by curettage from tubal infertility patients 21-39 years of age without EMs. RNA was extracted from a portion of the normal endometrial samples $(n=40)$ to detect the expression levels of hsa_circ_0001649, and ESCs were extracted from the remaining normal endometrial samples $(n=61)$ for subsequent experiments. Patients with pelvic inflammatory disease, adenomyosis and dysfunctional uterine bleeding were excluded. The participants had not received hormonal therapy for $\geq 6$ months before the surgical procedure. Informed consent was obtained with approval from the Local Ethics Committee of Tongji Medical College, Huazhong University of Science and Technology. All patients signed an informed consent form before entering the study. All samples were obtained during the proliferative phase of the regular menstrual cycle to avoid the effect of progesterone according to their last menstrual period and further confirmed histologically by Noyes criteria (Noyes \& Haman 1953, Noyes et al. 1975).

\section{Cell culture}

Human primary ESCs were isolated from the normal endometrium of patients with tubal infertility without EMs. The tissues were minced with scissors and digested enzymatically with collagenase type II (0.1\%; Sigma-Aldrich) for $40 \mathrm{~min}$ at $37^{\circ} \mathrm{C}$ with constant agitation. Then, the tissue pieces were sequentially filtered through sterile $400-\mu \mathrm{m}$ and $100-\mu \mathrm{m}$ sieve wires to remove undigested tissue and epithelial cells, and the filtrate was centrifuged at $129 \mathrm{~g}$ for 5 min to separate the ESCs from the collagenase. The ESC pellets were suspended in Red Blood Cell Lysis Buffer (C3702, Beyotime, Jiangsu, China) for $5 \mathrm{~min}$ to remove erythrocytes. The cell suspensions were centrifuged at $129 \mathrm{~g}$ for $5 \mathrm{~min}$, and the pelleted ESCs were resuspended in Dulbecco's Modified Eagle's Medium (DMEM)/F-12 medium containing 10\% fetal bovine serum (FBS). The ESCs were placed in a culture flask and incubated in $5 \% \mathrm{CO}_{2}$ at $37^{\circ} \mathrm{C}$.

A human endometrial stromal cell line (ThESCs) was purchased from American Type Culture Collection (CRL-4003; ATCC) and cultured in the same medium and environment as the ESCs.

\section{Hormone treatment}

$\mathrm{E}_{2}$ (E-2758, Sigma-Aldrich) and an $\mathrm{E}_{2}$ receptor (ER) antagonist ICI 182,780 (CAS 129453-61-8, Cayman Chemicals) were dissolved in DMSO. After incubation in serum-free and phenol red-free DMEM/F-12 for $24 \mathrm{~h}$, ESCs and ThESCs were treated with different concentrations $\left(0,10^{-11}, 10^{-10}, 10^{-9}, 10^{-8}\right.$, and $\left.10^{-7} \mathrm{~mol} / \mathrm{L}\right)$ of $\mathrm{E}_{2}$ and incubated for different durations $(0,12$, 24 , and $48 \mathrm{~h}$ ). The medium was changed to fresh complete medium every $24 \mathrm{~h}$.

\section{Quantitative RT-PCR (qRT-PCR)}

Total RNA was extracted from the tissue samples and cultured cells using TRIzol reagent (VazymeBiotech, Nanjing, China). cDNA was synthesized with HiScript II Q RT SuperMix (VazymeBiotech), qRT-PCR was performed using a StepOnePlus real-time PCR system (Applied Biosystems) with the preset PCR program, and GAPDH was used as an internal control to quantify mRNA expression. 
The qRT-PCR primer sequences are shown in Supplementary Table 1 (see section on supplementary materials given at the end of this article), where the different primers used to quantify hsa_circ_0001649 are cited from a study by Wang et al. (2018). The primers for hsa_circ_0001649 are located in the flanking region of the back-splice site where exon 26 and exon 29 are linked, as shown in Supplementary Fig. 1 (Zhong et al. 2018). The qRT-PCR products of hsa_circ_0001649 were analyzed by Sanger sequencing to confirm the back-splice junction of hsa_ circ_0001649. The PCR cycling conditions were $95^{\circ} \mathrm{C}$ for 30 $\mathrm{s}$, followed by $95^{\circ} \mathrm{C}$ for $10 \mathrm{~s}, 60^{\circ} \mathrm{C}$ for $30 \mathrm{~s}$, and a dissociation program of $95^{\circ} \mathrm{C}$ for $15 \mathrm{~s}, 60^{\circ} \mathrm{C}$ for $30 \mathrm{~s}$ and $95^{\circ} \mathrm{C}$ for $15 \mathrm{~s}$. The relative expression levels of the target genes were calculated by the $2^{-\Delta \Delta \mathrm{Ct}}$ method.

\section{Western blotting}

The total proteins extracted from the cultured ESCs and ThESCs were quantified with a BCA protein assay kit (P0010S; Beyotime). The protein samples were incubated for $10 \mathrm{~min}$ at $95^{\circ} \mathrm{C}$; then, proteins (30 mg) were subjected to $10 \%$ SDSPAGE and transferred to PVDF membranes $(0.45-\mu \mathrm{m}$ pore size; Millipore). The blots were incubated with $5 \%$ skim milk in Tris-buffered saline containing 0.05\% Tween 20 at room temperature for $1 \mathrm{~h}$ and then incubated overnight at $4^{\circ} \mathrm{C}$ with rabbit polyclonal anti-MMP9 (1:1000; 10375-2-AP; Proteintech, Rosemont, Illinois, USA) or rabbit monoclonal anti-GAPDH (1:1000; ab181602; Abcam) primary antibodies. Subsequently, the membranes were washed with TBST and incubated with a secondary anti-rabbit antibody (1:4000; Affinity, USA) for $1 \mathrm{~h}$ at room temperature. The proteins were visualized by the enhanced chemiluminescence method (WBKLS0500; Millipore) according to the manufacturer's recommendations.

\section{Hsa_circ_0001649-knockdown plasmid construction}

ShRNA against hsa_circ_0001649 (GenePharma, Shanghai, China) was designed to target the hsa circ_0001649 BSJ region and cloned into a pGPU6-GFPNeo vector for use. The shRNA sequences were as follows: 5'-CACCGTGGCTGCCСTTCTCTCAGCTTCAAGAGAGCTG AGAGAAGGGCAGCCATTTTTTG-3' (sense) and 5'-GATCCA AAAAATGGCTGCССТTCTCTCAGCTCTCTTGAAG CTGAGAGAAGGGCAGCCAC-3' (antisense).

ShRNA for hsa_circ_0001649 was synthesized in the nucleus after being transfected into cells and subsequently transported to the cytoplasm. With the Dicer-containing complex, the stem-loop structure in the shRNA was removed and siRNA was formed. The antisense strand of the siRNA was then incorporated into the RNA-induced silencing complex (RISC) after chain unwinding. Hsa_circ_0001649 was cleaved by the RISC, with the siRNA binding to the BSJ region in a base complementary manner, and subsequently degraded (as shown in Supplementary Fig. 2A). Control shRNA was constructed with the same vector as the hsa_circ_0001649 shRNA construct and sequences that are not complementary to the BSJ region. In addition, these sequences have been proven to have no effect on normal human cells (Cheng et al. 2010, Li et al.
2013, Wang et al. 2015). The shNC sequences were as follows: 5'-CACCGTTCTCCGAACGTGTCACGTCAAGAGATTACGT GACACGTTCGGAGAA TTTTTTG-3' (sense) and 5'-GATCCAAAAAATTCTCCGAACGTGTCACGT AATCTCTTG ACGTGACACGTTCGGAGAAC-3' (antisense) (as shown in Supplementary Fig. 2B).

\section{Hsa_circ_0001649-overexpression plasmid construction}

A pcDNA3.1 vector that can effectively overexpress circRNA was used for hsa_circ_0001649 overexpression (Liang \& Wilusz 2014), and an empty plasmid vector was used as a control (GenePharma). CircRNA overexpression vectors include two reverse complementary sequences (an upstream fragment and a downstream fragment, as shown in Supplementary Fig. 3) and the circRNA sequence. The upstream and downstream reverse complementary sequences produce a stable RNA hairpin structure after transcription to promote circRNA circularization. The full-length hsa_circ_0001649 sequence was inserted between the reverse complementary sequences with restriction enzyme cutting sites (Nhel, HindIII) and linked to the vector to construct an hsa_circ_0001649-overexpression vector (Supplementary Fig. 3). The overexpression efficiency of hsa_circ_0001649 was examined by qRT-PCR.

\section{Cell transfection}

ThESCs were seeded in six-well plates, grown to $60-80 \%$ confluence, and transfected with the indicated plasmids using Neofect (Neofect Biotech, Beijing, China). The cells were transfected for $48 \mathrm{~h}$ and then collected for subsequent analyses.

\section{Transwell assays}

Transwell units (24-well plates, insert membranes with $8-\mu \mathrm{m}$ pores; Corning Costar) were coated with or without Matrigel to investigate the cell migration and invasion capacities, respectively. In each well, transfected ThESCs were resuspended in $200 \mu \mathrm{L}$ of serum-free DMEM/F12 medium with or without $17 \beta-E_{2}$ and placed in the upper chamber, and 500 $\mu \mathrm{L}$ of complete medium was added to the lower chamber. The transwell units were incubated in $5 \% \mathrm{CO}_{2}$ at $37^{\circ} \mathrm{C}$ for 24 $h$, and the cells and Matrigel were removed from the upper membrane surface and stained with $0.1 \%$ crystal violet for $20 \mathrm{~min}$ at $37^{\circ} \mathrm{C}$. The number of cells on the underside of the membrane was counted under a light microscope (Olympus). Five randomly selected fields were counted per insert.

\section{Statistical analysis}

All data are shown as the mean \pm S.D. Statistical analyses were performed using GraphPad Prism 5.01 software (GraphPad Software) and SPSS 19.0 statistical software (SPSS). The Kruskal-Wallis test was used to compare non-normally distributed variables. For normally distributed variables, an unpaired Student's $t$-test was used to determine significant differences between two groups, and one-way ANOVA was 
used for comparisons between three or more groups. $P<0.05$ was considered statistically significant. All data were obtained from $\geq 3$ independent experiments.

\section{Results}

\section{Characterization and expression of hsa_circ_0001649 in EMs}

To determine whether hsa_circ_0001649 is involved in the pathogenesis of EMs, we acquired the hsa circ_0001649 products amplified by qRT-PCR, which were used for Sanger sequencing, and the result was consistent with the sequence in CircBase (Fig. 1A), revealing the expression of hsa_circ_0001649 in EMs tissue. Then, we measured the relative expression of hsa_circ_0001649 in 40 paired eutopic and ectopic endometrial samples from patients with EMs and 40 normal endometrial samples by qRT-PCR. As indicated in Fig. 1B, compared with that in the normal endometrium, the expression level of hsa_circ_0001649 was significantly decreased in ectopic $(P<0.0001)$ and eutopic $(P<0.0001)$ endometrium samples from EMs patients.
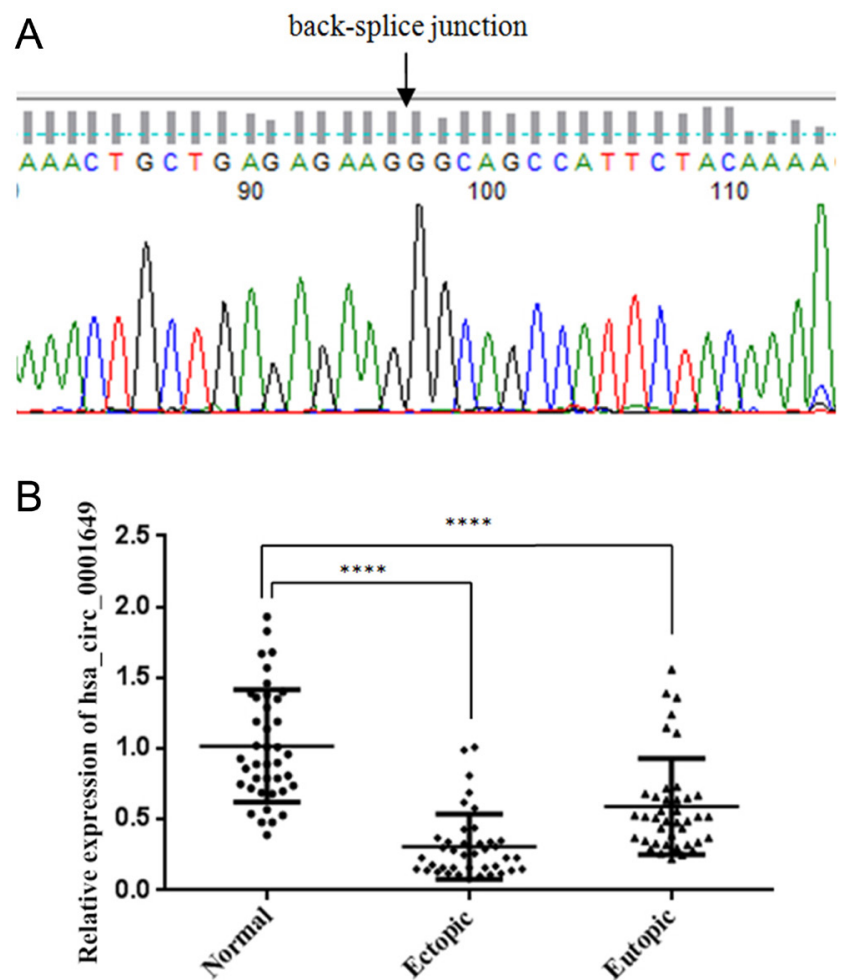

Figure 1 Characterization and expression of hsa_circ_0001649 in EMs. (A) The Sanger sequence of hsa_circ_0001649 qRT-PCR products. (B) The relative expression levels of hsa_circ_0001649 in eutopic $(n=40)$ and ectopic $(n=40)$ endometrial specimens from patients with ovarian endometriosis (EMs) and normal endometrial $(n=40)$ specimens were measured by qRT-PCR. The data are expressed as the mean \pm S.D. ${ }^{* * * *} P<0.0001$ by the Kruskal-Wallis test.

\section{$E_{2}$ downregulates hsa_circ_0001649 through estrogen receptors (ERs) in ESCs and ThESCs}

As $E M s$ is an $E_{2}$-dependent disease, we investigated whether the lower hsa_circ_0001649 expression in EMs patients than that in the normal endometrium is caused by $E_{2}$. Both ESCs and ThESCs were treated with various gradient concentrations of $17 \beta-\mathrm{E}_{2}\left(0,10^{-11}, 10^{-10}\right.$, $10^{-9}, 10^{-8}$, and $\left.10^{-7} \mathrm{~mol} / \mathrm{L}\right)$ for $48 \mathrm{~h}$. We observed that $\mathrm{E}_{2}$ decreased hsa_circ_0001649 expression in ESCs and ThESCs in a concentration-dependent manner, with significant inhibition at $10^{-8} \mathrm{~mol} / \mathrm{L}$ and $10^{-7}$ $\mathrm{mol} / \mathrm{L}(P<0.0001)$ (Fig. $2 \mathrm{~A}$ and B). The physiological concentration of $\mathrm{E}_{2}$ in women ranges from approximately $10^{-10} \mathrm{~mol} / \mathrm{L}$ to $10^{-9} \mathrm{~mol} / \mathrm{L}$, whereas the endometriotic intra tissue $\mathrm{E}_{2}$ concentration is approximately $10^{-8} \mathrm{~mol} / \mathrm{L}$ (Huhtinen et al. 2012). We compared hsa_circ_0001649 expression in cells treated with $10^{-9} \mathrm{~mol} / \mathrm{L} \mathrm{E}_{2}$ with that
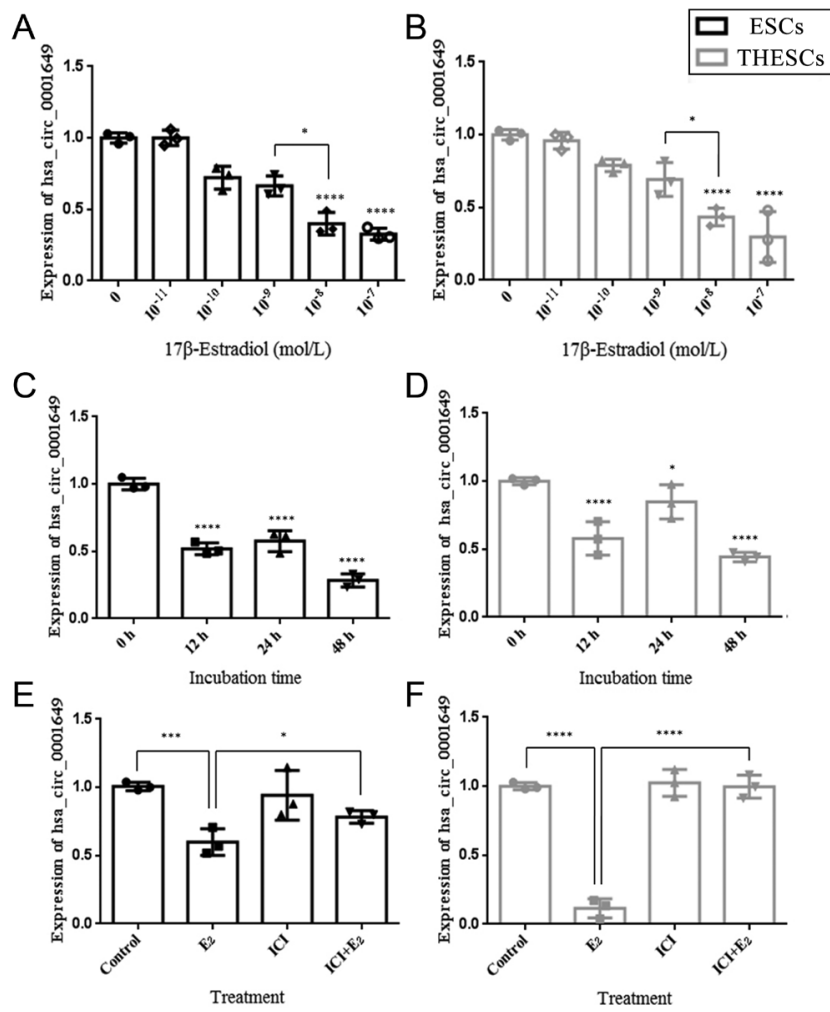

Figure 2 Hsa_circ_0001649 expression is regulated by estrogen (E2) in human primary endometrial stromal cells (ESCs) and a human endometrial stromal cell line (ThESCs). (A and B) Hsa_circ_0001649 expression in ESCs and ThESCs was determined by qRT-PCR following treatment with $E_{2}$ at a series of concentrations $\left(0,10^{-11}\right.$, $10^{-10}, 10^{-9}, 10^{-8}$, and $10^{-7} \mathrm{~mol} / \mathrm{L}$ ). (C and D) Hsa_circ_0001649 expression in ESCs and ThESCs was determined following $10^{-8} \mathrm{~mol} / \mathrm{L}$ $\mathrm{E}_{2}$ treatment for the indicated durations $(0,12,24$, and $48 \mathrm{~h})$. ( $\mathrm{E}$ and F) Hsa_circ_0001649 expression was determined following vehicle, $10^{-8} \mathrm{~mol} / \mathrm{L} \mathrm{E}_{2}, 10^{-6} \mathrm{~mol} / \mathrm{L}$ estrogen receptor antagonist ICI 182,780 $(\mathrm{ICl})$ and $10^{-8} \mathrm{~mol} / \mathrm{L} \mathrm{E}_{2}+10^{-6} \mathrm{~mol} / \mathrm{L} \mathrm{ICl}$ stimulation for $48 \mathrm{~h}$. The data are expressed as the mean \pm S.D. of three independent experiments. ${ }^{*} P<0.05 ;{ }^{* * *} P<0.001$ and ${ }^{* * * *} P<0.0001$ by one-way ANOVA. 
in cells treated with $10^{-8} \mathrm{~mol} / \mathrm{L} \mathrm{E}_{2}$ and found significant decreases in cells under $10^{-8} \mathrm{~mol} / \mathrm{L} \quad E_{2}$ stimulation $(P<0.05)$ (Fig. 2A and B). Therefore, together with the results obtained from the concentration gradient experiments, we selected $10^{-8} \mathrm{~mol} / \mathrm{L} \mathrm{E}_{2}$ stimulation for our subsequent experiments. ESCs and ThESCs were cultured with $10^{-8} \mathrm{~mol} / \mathrm{L} \mathrm{E}_{2}$ for different durations $(0,12$, 24 , or 48 h). As shown in Fig. 2C, compared with that in unstimulated control cells, a time-dependent decrease in hsa_circ_0001649 expression was observed, and the lowest level was observed at $48 \mathrm{~h}(P<0.0001)$. Similar results were obtained with the ThESCs, with a significant reduction in the expression of hsa_circ_0001649 being observed at $48 \mathrm{~h}(P<0.0001)$ (Fig. 2D).

To further determine whether the $\mathrm{E}_{2}$-mediated regulation of hsa_circ_0001649 expression was dependent on ERs, ESCs and ThESCs were divided into the following four experimental groups: group 1, control group (no treatment); group 2, treated with $10^{-8} \mathrm{~mol} / \mathrm{L} \mathrm{E}_{2}$; group 3, treated with $10^{-6} \mathrm{~mol} / \mathrm{L} \mathrm{ICl}$; and group 4, treated with $10^{-6} \mathrm{~mol} / \mathrm{L} \mathrm{ICl}$ in conjunction with $10^{-8} \mathrm{~mol} / \mathrm{L} \mathrm{E}_{2}$. As shown in Fig. $2 \mathrm{E}$ and $\mathrm{F}$, the $\mathrm{E}_{2}$-mediated inhibition of hsa_circ_0001649 expression was antagonized by $\mathrm{ICI}$ in the ESCs $(P<0.05)$ and ThESCs $(P<0.0001)$. These results suggest that $E_{2}$ inhibits the expression of hsa circ_0001649 in ESCs and ThESCs via ERs.

\section{$E_{2}$ increases MMP9 expression in ESCs and ThESCs through hsa_circ_0001649}

To investigate the action of $E_{2}$ on MMP9, ESCs and ThESCs were treated with $\mathrm{E}_{2}, \mathrm{ICl}$, and $\mathrm{ICl}$ in conjunction with $E_{2}$ and compared with vehicle-only controls. Western blot and qRT-PCR analyses demonstrated that
A

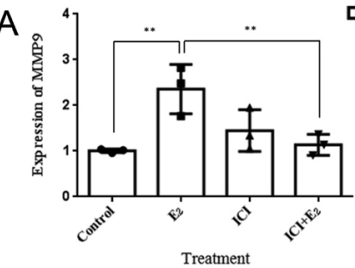

口 $\mathrm{ESCS}$

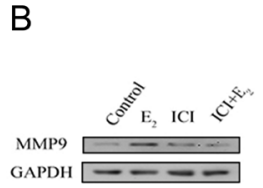

C

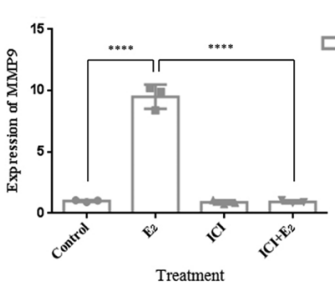

$\square$ THESC

D
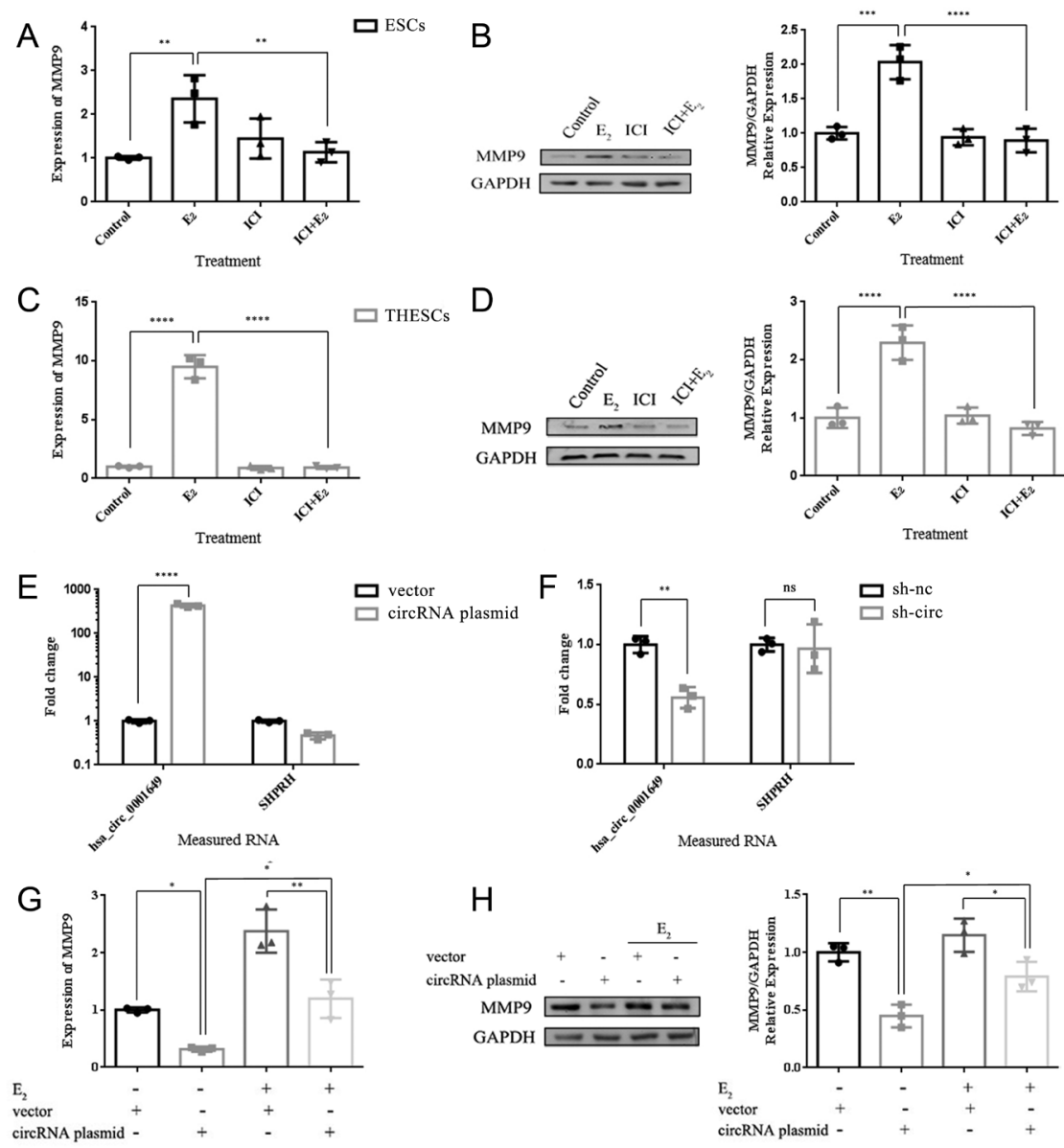

I

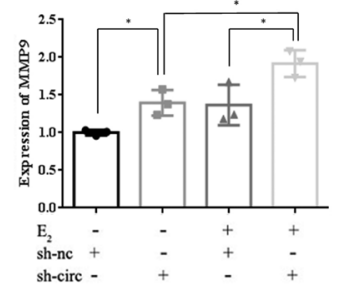

$J$
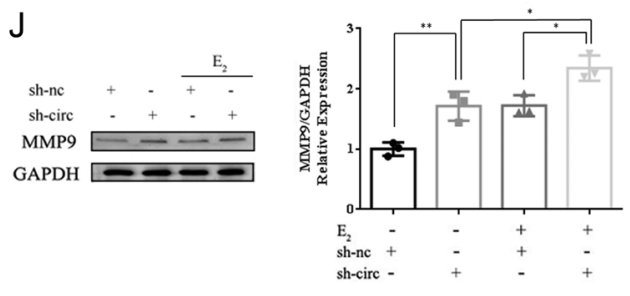

Figure 3 Effects of hsa_circ_0001649 on estrogen $\left(E_{2}\right)$-mediated MMP9 expression in ESCs and ThESCs. (A, B, C and D) MMP9 mRNA and protein expression in ESCs and ThESCs treated with vehicle-only controls, $E_{2}$, $\mathrm{ICl}$ and $\mathrm{E}_{2}+\mathrm{ICl}$. (E) hsa_circ_0001649 and SHPRH (negative control) expression in ThESCs transfected with a pcDNA3.1 vector overexpressing hsa_circ_0001649 (circRNA plasmid) or an empty vector (vector). (F) hsa_circ_0001649 and SHPRH expression in ThESCs transfected with hsa_circ_0001649 shRNA (sh-circ) or control shRNA (sh-nc). (G and $\mathrm{H}$ ) MMP9 mRNA and protein expression in ThESCs transfected with vector, circRNA plasmid, vector $+E_{2}$, and circRNA plasmid $+E_{2}$. (I and J) MMP9 mRNA and protein expression in ThESCs transfected with sh-nc, sh-circ, sh-nc $+E_{2}$, and sh-circ $+E_{2}$. RNA expression was measured by qRT-PCR and protein expression by Western blotting; the results are represented as the fold change compared to the vehicle-only controls. The data are presented as the mean \pm S.D. of three independent experiments. ${ }^{*} P<0.05$;

${ }^{* *} P<0.01$; ${ }^{* * *} P<0.0001$; and ${ }^{* * * *} P<0.0001$ by one-way ANOVA and Student's t-test. n.s., not significant. 
$\mathrm{E}_{2}$-mediated expression of MMP9 was antagonized by ICI at the protein and mRNA levels in both ESCs $(P<0.01)$ (Fig. $3 \mathrm{~A}$ and $\mathrm{B})$ and ThESCs $(P<0.0001)$ (Fig. 3C and D). Altogether, these results indicate that $E_{2}$ increases MMP9 expression via ERs.

Then, we further investigated whether hsa circ_0001649 is involved in $\mathrm{E}_{2}$-mediated MMP9 expression. Hsa_circ_0001649 expression in ThESCs was effectively knocked down by the shRNA specifically targeting the splice junction of hsa_circ_0001649 (sh-circ) or increased by an hsa_circ_0001649-forming plasmid (circRNA plasmid) (Fig. 3E and F). qRT-PCR and Western blot analyses showed that knocking down the expression of hsa_circ_0001649 under the $\mathrm{E}_{2}$ conditions resulted in the maximum MMP9 expression, while the overexpression of hsa_circ_0001649 not only inhibited MMP9 expression but also reversed the promotional effect of $\mathrm{E}_{2}$ on MMP9 (Fig. 3G, $\mathrm{H}, \mathrm{I}$ and J). These results suggest that hsa_circ_0001649 is involved in $\mathrm{E}_{2}$ mediated MMP9 expression.

\section{Hsa_circ_0001649 influences the ThESC migration and invasion capacities}

Considering the downregulation of hsa_circ_0001649 in EMs and its relationship with MMP9, determining its functional role was necessary. Therefore, transwell assays were used to detect the invasion and migration abilities of ThESCs. As shown in Fig. 4, $\mathrm{E}_{2}$ promoted the migration and invasion abilities of ThESCs, while overexpression of hsa_circ_0001649 reduced the migration and invasion of ThESCs and even reversed the promotional ability of $\mathrm{E}_{2}$ (Fig. 4A). Correspondingly, the knockdown of hsa circ_0001649 had the opposite effect (Fig. 4B). These results shed light on the metastasis-promoting role of hsa_circ_0001649 downregulation in ThESCs.

\section{Discussion}

In the present study, we evaluated hsa_circ_0001649 expression in women with EMs and found that,
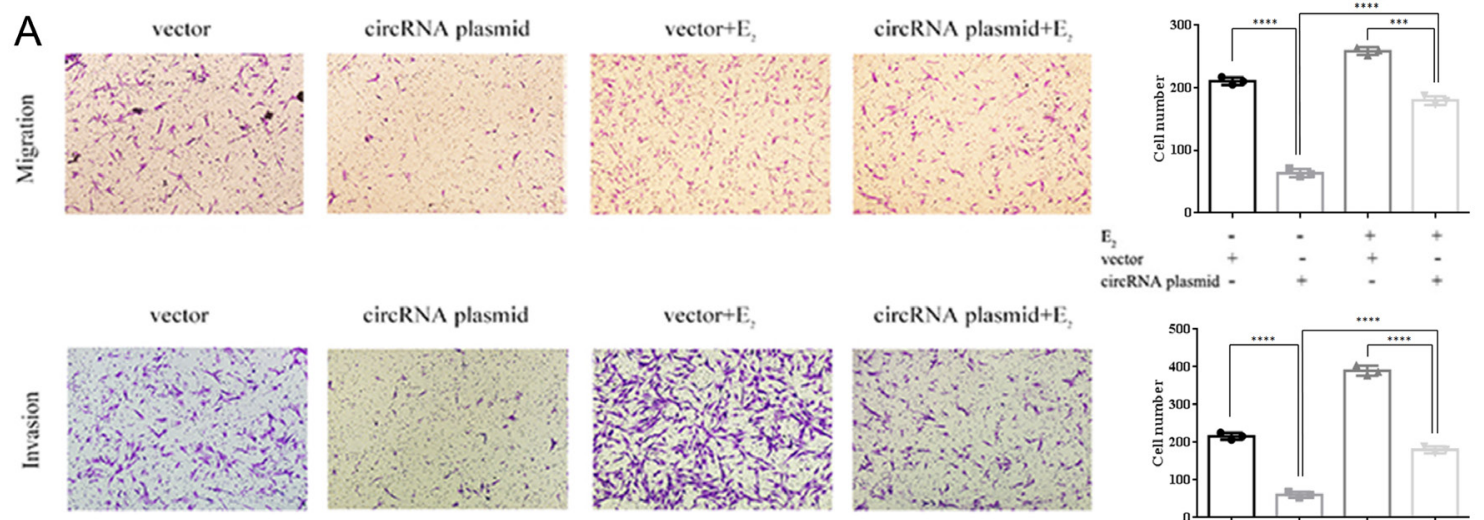

circRNA plasmid

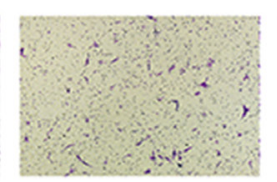

vector $+\mathrm{E}_{2}$
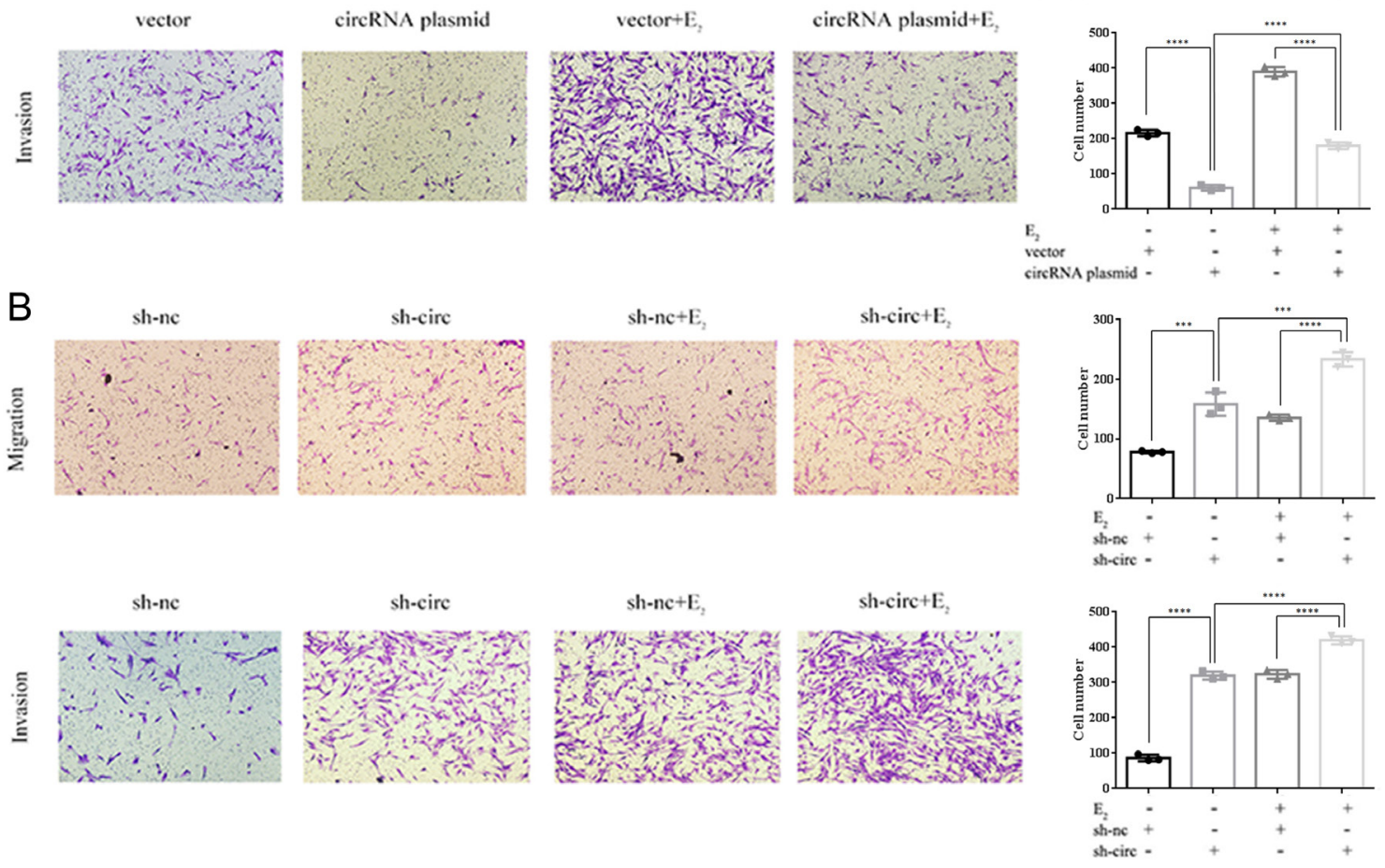

Figure 4 Hsa_circ_0001649 influences the migration and invasion capacities of ThESCs. (A) Transwell assays showed the migration and invasion capacities of ThESCs transfected with vector, circRNA plasmid, vector $+E_{2}$, and circRNA plasmid $+E_{2}$. (B) Transwell assays showed the migration and invasion capacities of ThESCs transfected with sh-nc, sh-circ, sh-nc $+E_{2}$, and sh-circ $+E_{2}$. All images were taken at 200x magnification. The data are presented as the mean \pm S.D. of three independent experiments. ${ }^{* * *} P<0.0001$ and ${ }^{* * * *} P<0.0001$ by one-way ANOVA. 
compared with normal endometrial tissue, ectopic and eutopic endometrial tissues from EMs patients had lower hsa_circ_0001649 levels. Moreover, hsa_circ_0001649 was involved in the $E_{2}$-mediated upregulation of MMP9 in ESCs and ThESCs, suggesting that hsa_circ_0001649 functions as a migration-invasion suppressor in EMs, which is consistent with its function in colorectal cancer, gastric cancer and hepatocellular carcinoma (Qin et al. 2016, Li et al. 2017a, Ji et al. 2018).

CircRNAs represent newly discovered star RNAs. Currently, research investigating circRNAs is insufficient and many problems remain to be solved. Two outstanding problems include the identification of factors that regulate circRNAs and the mechanism by which circRNAs exert physiological and pathological effects. Therefore, our finding of differential hsa_circ_0001649 expression in EMs represents an initial step toward identifying the factors underlying decreased hsa_circ_0001649 expression. Studies have shown that, in clear cell renal cell carcinoma, breast cancer and granulosa cells, the expression levels of circRNAs are associated with $E_{2}$ or Ers (Huang et al. 2018, Klinge 2018, Qiao et al. 2018). Because $E_{2}$ plays key roles in Ems (Huhtinen et al. 2012), we suspected and further confirmed experimentally that $E_{2}$ can inhibit circRNA expression in ESCs through ERs. However, further studies are needed to determine which ER plays a role in the regulatory mechanism. Although most studies have reported changes only in downstream circRNA expression during $E_{2}$ action or ER knockout (Nair et al. 2016, Li et al. 2017b, Song et al. 2019, Yuan et al. 2019), one study showed that, in bladder cancer, ER $\alpha$ can regulate the expression of circ_0023642 by regulating the expression of its host gene UVRAG at the transcriptional level (Wu et al. 2019). We used JASPAR (http://jaspar.genereg.net/), a web-based approach, to predict potential $E_{2}$ response elements (EREs) in the promoter region of the hsa circ_0001649 host gene SHPRH and identified several potential ER $\beta$ binding sites. ChIP and dual-luciferase assays can be used to verify whether ER $\beta$ has a direct regulatory effect on SHPRH. Moreover, the RNA-binding protein Quaking $(\mathrm{QKI})$ regulates circRNA formation during EMT by binding sites flanking circRNA-forming exons and inducing exon circularization (Conn et al. 2015). Notably, hsa_circ_0001649 is strongly regulated following EMT (Conn et al. 2015), which indicates that QKI directly binds to the host gene SHPRH to promote hsa_circ_0001649 formation in EMs. Moreover, the information in the JASPAR database shows that QKI does not have an ER $\alpha$ binding site, but it does have an ER $\beta$ binding site in the upstream promoter region, suggesting that it may be regulated by ER $\beta$. Studies have reported that higher ER $\beta$ levels and enhanced ER $\beta$ activity are detected in endometriotic tissues (Han et al. 2015). Therefore, whether ER $\beta$ can regulate QKI or directly binds to SHPRH to reduce hsa_circ_0001649 expression in EMs is worthy of further investigation.
We next studied the function of hsa_circ_0001649 and revealed that it increases MMP9 expression and affects invasion and metastasis in ESCs, but we did not further study the specific mechanism by which hsa_circ_0001649 impacts MMP9. Furthermore, the functions of circRNAs remain largely unknown. It has been proposed that circRNAs may act as competing endogenous RNAs (ceRNA) to miRNAs (miRNA sponges). The two most widely known examples are ciRS-7/CDR1as, which functions as a miR-7 sponge, and sex-determining region $\mathrm{Y}$ (Sry) 9, which functions as a miR-138 sponge (Huhtinen et al. 2012, Klinge 2018). We found that hsa_circ_0001649 has the capacity to harbor miRNA binding sites and possesses potential binding sites for hsa-miR-1231, hsa-miR-223 and 20 other miRNAs (https://circinteractome.nia.nih.gov/ miRNA_Target_Sites/mirna_target_sites.html). Based on Circlnteractome (a web tool that can explore potential miRNAs interacting with circRNAs), we found that hsa_circ_0001649 has the capacity to harbor miRNA binding sites and possesses potential binding sites for hsa-miR-1231, hsa-miR-223 and 19 other miRNAs. Further prediction based on TargetScan (miRNA target prediction software that uses the same algorithm as Circlnteractome) revealed that hsa_circ_0001649 has four binding sites for hsa-miR-203a, two binding sites for hsa-miR-127, hsa-miR-331, hsa-miR-486, hsa-miR-488, hsa-miR-545, hsa-miR-649, and hsa-miR-942 and one binding site for 13 others miRNAs (Supplementary Table 2) (Dudekula et al. 2016). Therefore, hsa_circ_0001649 might play a role in EMs through interactions with miRNAs. However, other studies have suggested that few circRNAs contain a substantial number of miRNA binding sites for a single miRNA, and it remains to be clarified whether circRNA can function as a miRNA sponge (Ebbesen et al. 2016). A search for reiterated miRNA binding sites in circRNAs regulated by transforming growth factor $\beta$ treatment inhuman mammary epithelial cells did not reveal any candidates (Conn et al. 2015). Similarly, a computational analysis

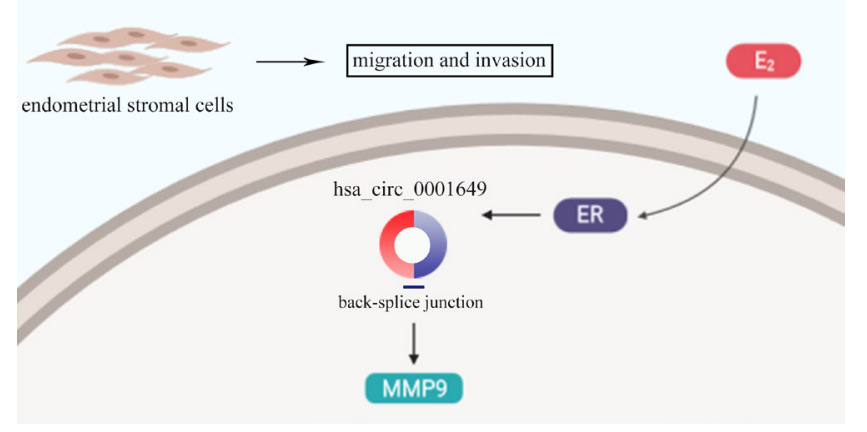

Figure 5 A schematic diagram depicting how estrogen affects migration and invasion by regulating hsa_circ_0001649 expression in human primary endometrial stromal cells (ESCs). The image was created with BioRender.com. 
of available circRNA sequences identified only two with more miRNA sites than expected by chance (Guo et al. 2014). Thus, RNA pull-down and other experiments are needed to further elucidate the mechanism of hsa circ_0001649 and determine whether it functions as a miRNA sponge in EMs.

In summary, our results show that $E_{2}$ decreased hsa circ_0001649 expression through ERs, which further upregulated the expression of MMP9 and enhanced ESC migration and invasion (Fig. 5). These findings have improved our understanding of the molecular mechanisms underlying $\mathrm{E}_{2}$-mediated cell invasion in EMs.

\section{Supplementary materials}

This is linked to the online version of the paper at https://doi. org/10.1530/REP-19-0540.

\section{Declaration of interest}

The authors declare that there is no conflict of interest that could be perceived as prejudicing the impartiality of the research reported.

\section{Funding}

This project was supported by the National Natural Science Foundation of China (Grant No. 81471439 Y L) and by Hubei Provincial Natural Science Foundation of China (2019CFB148).

\section{Author contribution statement}

Q Li, Y Liu and L Zhang conceived the study and wrote the paper. N Li, H Liu, Y Du and H He performed the experiments and analyzed the data.

\section{References}

American Society for Reproductive Medicine 1997 Revised American society for reproductive medicine classification of endometriosis: 1996. Fertility and Sterility 67 817-821. (https://doi.org/10.1016/s00150282(97)81391-x)

Anastasiadou E, Jacob LS \& Slack FJ 2018 Non-coding RNA networks in cancer. Nature Reviews: Cancer 18 5-18. (https://doi.org/10.1038/ nrc.2017.99)

Cech TR \& Steitz JA 2014 The noncoding RNA revolution-trashing old rules to forge new ones. Cell 157 77-94. (https://doi.org/10.1016/j. cell.2014.03.008)

Cheng S, Zhang J, Zhu P, Ma Y, Xiong Y, Sun L, Xu J, Zhang H \& He J 2010 The PDZ domain protein CAL interacts with mGluR5a and modulates receptor expression. Journal of Neurochemistry 112 588-598. (https:// doi.org/10.1111/j.1471-4159.2009.06454.x)

Conn SJ, Pillman KA, Toubia J, Conn VM, Salmanidis M, Phillips CA, Roslan S, Schreiber AW, Gregory PA \& Goodall GJ 2015 The RNA binding protein quaking regulates formation of circRNAs. Cell $\mathbf{1 6 0}$ 1125-1134. (https://doi.org/10.1016/j.cell.2015.02.014)

Du Y, Zhang Z, Xiong W, Li N, Liu H, He H, Li Q, Liu Y \& Zhang L 2019 Estradiol promotes EMT in endometriosis via MALAT1/miR200s sponge function. Reproduction 157 179-188. (https://doi.org/10.1530/REP-180424)
Dudekula DB, Panda AC, Grammatikakis I, De S, Abdelmohsen K \& Gorospe M 2016 Circlnteractome: a web tool for exploring circular RNAs and their interacting proteins and microRNAs. RNA Biology 13 34-42. (https://doi.org/10.1080/15476286.2015.1128065)

Ebbesen KK, Kjems J \& Hansen TB 2016 Circular RNAs: identification, biogenesis and function. Biochimica et Biophysica Acta 1859 163-168. (https://doi.org/10.1016/j.bbagrm.2015.07.007)

Esteller M 2011 Non-coding RNAs in human disease. Nature Reviews: Genetics 12 861-874. (https://doi.org/10.1038/nrg3074)

Ferlita A, Battaglia R, Andronico F, Caruso S, Cianci A, Purrello M \& Pietro CD 2018 Non-coding RNAs in endometrial physiopathology. International Journal of Molecular Sciences 19 E2120. (https://doi. org/10.3390/ijms19072120)

Greene J, Baird AM, Brady L, Lim M, Gray SG, McDermott R \& Finn SP 2017 Circular RNAs: biogenesis, function and role in human diseases. Frontiers in Molecular Biosciences 4 38. (https://doi.org/10.3389/ fmolb.2017.00038)

Guo JU, Agarwal V, Guo H \& Bartel DP 2014 Expanded identification and characterization of mammalian circular RNAs. Genome Biology 15409. (https://doi.org/10.1186/s13059-014-0409-z)

Han SJ, Jung SY, Wu SP, Hawkins SM, Park MJ, Kyo S, Qin J, Lydon JP, Tsai SY, Tsai MJ et al. 2015 Estrogen receptor beta modulates apoptosis complexes and the inflammasome to drive the pathogenesis of endometriosis. Cell 163 960-974. (https://doi.org/10.1016/j. cell.2015.10.034)

Hansen TB, Jensen TI, Clausen BH, Bramsen JB, Finsen B, Damgaard CK \& Kjems J 2013 Natural RNA circles function as efficient microRNA sponges. Nature 495 384-388. (https://doi.org/10.1038/nature11993)

Harada T, Iwabe T \& Terakawa N 2001 Role of cytokines in endometriosis. Fertility and Sterility 76 1-10. (https://doi.org/10.1016/s00150282(01)01816-7)

Huang BH, Chang CW, Huang CW, Gao J \& Liao PC 2018 Composition and functional specialists of the gut microbiota of frogs reflect habitat differences and agricultural activity. Frontiers in Microbiology 82670. (https://doi.org/10.3389/fmicb.2017.02670)

Huhtinen K, Stahle M, Perheentupa A \& Poutanen M 2012 Estrogen biosynthesis and signaling in endometriosis. Molecular and Cellular Endocrinology 358 146-154. (https://doi.org/10.1016/j.mce.2011.08.022)

Ji W, Qiu C, Wang M, Mao N, Wu S \& Dai Y 2018 Hsa_circ_0001649: a circular RNA and potential novel biomarker for colorectal cancer. Biochemical and Biophysical Research Communications 497 122-126. (https://doi.org/10.1016/j.bbrc.2018.02.036)

Klinge CM 2018 Non-coding RNAs in breast cancer: intracellular and intercellular communication. Non-Coding RNA 4 E40. (https://doi. org/10.3390/ncrna4040040)

Kodaman PH 2015 Current strategies for endometriosis management. Obstetrics and Gynecology Clinics of North America 42 87-101. (https:// doi.org/10.1016/j.ogc.2014.10.005)

Kvaskoff M, Mu F, Terry KL, Harris HR, Poole EM, Farland L \& Missmer SA 2015 Endometriosis: a high-risk population for major chronic diseases? Human Reproduction Update 21 500-516. (https://doi.org/10.1093/ humupd/dmv013)

Li WH, Song YC, Zhang H, Zhou ZJ, Xie X, Zeng QN, Guo K, Wang T, Xia P \& Chang DM 2017a Decreased expression of Hsa_circ_00001649 in gastric cancer and its clinical significance. Disease Markers 2017 4587698. (https://doi.org/10.1155/2017/4587698)

Li X, Peng B, Zhu X, Wang P, Xiong Y, Liu H, Sun K, Wang H, Ou L, Wu Z et al. 2017b Changes in related circular RNAs following ERbeta knockdown and the relationship to rBMSC osteogenesis. Biochemical and Biophysical Research Communications 493 100-107. (https://doi. org/10.1016/j.bbrc.2017.09.068)

Li XJ, Luo Y \& Yi YF 2013 P115 promotes growth of gastric cancer through interaction with macrophage migration inhibitory factor. World Journal of Gastroenterology 19 8619-8629. (https://doi.org/10.3748/wjg.v19. i46.8619)

Li Z, Huang C, Bao C, Chen L, Lin M, Wang X, Zhong G, Yu B, Hu W, Dai L et al. 2015 Exon-intron circular RNAs regulate transcription in the nucleus. Nature Structural and Molecular Biology 22 256-264. (https:// doi.org/10.1038/nsmb.2959)

Liang D \& Wilusz JE 2014 Short intronic repeat sequences facilitate circular RNA production. Genes and Development 28 2233-2247. (https://doi. org/10.1101/gad.251926.114) 
Liu H, Zhang Z, Xiong W, Zhang L, Du Y, Liu Y \& Xiong X 2019 Long noncoding RNA MALAT1 mediates hypoxia-induced pro-survival autophagy of endometrial stromal cells in endometriosis. Journal of Cellular and Molecular Medicine 23 439-452. (https://doi.org/10.1111/jcmm.13947)

Nair AA, Niu N, Tang X, Thompson KJ, Wang L, Kocher JP, Subramanian S \& Kalari KR 2016 Circular RNAs and their associations with breast cancer subtypes. Oncotarget 7 80967-80979. (https://doi.org/10.18632/ oncotarget.13134)

Noyes RW \& Haman JO 1953 Accuracy of endometrial dating; correlation of endometrial dating with basal body temperature and menses. Fertility and Sterility 4 504-517. (https://doi.org/10.1016/s0015-0282(16)31446-7)

Noyes RW, Hertig AT \& Rock J 1975 Dating the endometrial biopsy. American Journal of Obstetrics and Gynecology 122 262-263. (https:// doi.org/10.1016/s0002-9378(16)33500-1)

Pamudurti NR, Bartok O, Jens M, Ashwal-Fluss R, Stottmeister C, Ruhe L, Hanan M, Wyler E, Perez-Hernandez D, Ramberger E et al. 2017 Translation of CircRNAs. Molecular Cell 66 9.e7-21.e7. (https://doi. org/10.1016/j.molcel.2017.02.021)

Panir K, Schjenken JE, Robertson SA \& Hull ML 2018 Non-coding RNAs in endometriosis: a narrative review. Human Reproduction Update 24 497-515. (https://doi.org/10.1093/humupd/dmy014)

Pino M, Galleguillos C, Torres M, Sovino H, Fuentes A, Boric MA \& Johnson MC 2009 Association between MMP1 and MMP9 activities and ICAM1 cleavage induced by tumor necrosis factor in stromal cell cultures from eutopic endometria of women with endometriosis. Reproduction 138 837-847. (https://doi.org/10.1530/REP-09-0196)

Qiao F, Lei K, Li Z, Wei Z, Liu Q, Yang L, He J, An L, Qi H \& Cui S 2018 Transcriptomic responses of the freshwater snail (Parafossarulus striatulus) following dietary exposure to Cyanobacteria. Science of the Total Environment 624 153-161. (https://doi.org/10.1016/j. scitotenv.2017.12.112)

Qin M, Liu G, Huo X, Tao X, Sun X, Ge Z, Yang J, Fan J, Liu L \& Qin W 2016 Hsa_circ_0001649: a circular RNA and potential novel biomarker for hepatocellular carcinoma. Cancer Biomarkers: Section A of Disease Markers 16 161-169. (https://doi.org/10.3233/CBM-150552)

Qu S, Yang X, Li X, Wang J, Gao Y, Shang R, Sun W, Dou K \& Li H 2015 Circular RNA: a new star of noncoding RNAs. Cancer Letters 365 141-148. (https://doi.org/10.1016/j.canlet.2015.06.003)

Shen L, Zhang Y, Zhou W, Peng Z, Hong X \& Zhang Y 2018 Circular RNA expression in ovarian endometriosis. Epigenomics 10 559-572. (https:// doi.org/10.2217/epi-2017-0079)

Song Y, Zhang L, Liu X, Niu M, Cui J, Che S, Liu Y, An X \& Cao B 2019 Analyses of circRNA profiling during the development from pre-receptive to receptive phases in the goat endometrium. Journal of Animal Science and Biotechnology 10 34. (https://doi.org/10.1186/s40104-019-0339-4)

van Kempen LC \& Coussens LM 2002 MMP9 potentiates pulmonary metastasis formation. Cancer Cell 2 251-252. (https://doi.org/10.1016/ s1535-6108(02)00157-5)

Wang D, Luo Y, Wang G \& Yang Q 2019 Circular RNA expression profiles and bioinformatics analysis in ovarian endometriosis. Molecular Genetics and Genomic Medicine 7 e00756. (https://doi.org/10.1002/ mgg3.756)

Wang J, Wang Q, Han T, Li YK, Zhu SL, Ao F, Feng J, Jing MZ, Wang L, Ye LB et al. 2015 Soluble interleukin-6 receptor is elevated during influenza A virus infection and mediates the IL- 6 and IL-32 inflammatory cytokine burst. Cellular and Molecular Immunology 12 633-644. (https://doi. org/10.1038/cmi.2014.80)

Wang Y, Li L, Han Y, Liu J \& Yang K 2018 Intestinal bacteria in bioaerosols and factors affecting their survival in two oxidation ditch process municipal wastewater treatment plants located in different regions. Ecotoxicology and Environmental Safety 154 162-170. (https://doi. org/10.1016/j.ecoenv.2018.02.041)

Wu L, Zhang M, Qi L, Zu X, Li Y, Liu L, Chen M, Li Y, He W, Hu X et al. 2019 ERalpha-mediated alterations in circ_0023642 and miR-490-5p signaling suppress bladder cancer invasion. Cell Death and Disease 10 635. (https://doi.org/10.1038/s41419-019-1827-3)

Xing L, Zhang L, Feng Y, Cui Z \& Ding L 2018 Downregulation of circular RNA hsa_circ_0001649 indicates poor prognosis for retinoblastoma and regulates cell proliferation and apoptosis via AKT/mTOR signaling pathway. Biomedicine and Pharmacotherapy 105 326-333. (https://doi. org/10.1016/j.biopha.2018.05.141)

Xu X, Jia SZ, Dai Y, Zhang JJ, Li X, Shi J, Leng J \& Lang J $2018 b$ The relationship of circular RNAs with ovarian endometriosis. Reproductive Sciences 25 1292-1300. (https://doi.org/10.1177/1933719118759439)

Xu XX, Jia SZ, Dai Y, Zhang JJ, Li XY, Shi JH, Leng JH \& Lang JH 2018a Identification of circular RNAs as a novel biomarker for ovarian endometriosis. Chinese Medical Journal 131 559-566. (https://doi. org/10.4103/0366-6999.226070)

Yilmaz BD \& Bulun SE 2019 Endometriosis and nuclear receptors. Human Reproduction Update 25 473-485. (https://doi.org/10.1093/humupd/ dmz005)

Yuan C, Zhou L, Zhang L, Yin K, Peng J, Sha R, Zhang S, Xu Y, Sheng X, Wang $\mathbf{Y}$ et al. 2019 Identification and integrated analysis of key differentially expressed circular RNAs in ER-positive subtype breast cancer. Epigenomics 11 297-321. (https://doi.org/10.2217/epi-20180147)

Zang J, Lu D \& Xu A 2020 The interaction of circRNAs and RNA binding proteins: an important part of circRNA maintenance and function. Journal of Neuroscience Research 98 87-97. (https://doi.org/10.1002/ jnr.24356)

Zhang L, Xiong W, Xiong Y, Liu H, Li N, Du Y \& Liu Y 2016 Intracellular Wnt/beta-catenin signaling underlying 17beta-estradiol-induced matrix metalloproteinase 9 expression in human endometriosis. Biology of Reproduction $94 \quad 70 . \quad$ (https://doi.org/10.1095/ biolreprod.115.135574)

Zhang M, Ren C, Xiao Y, Xia X \& Fang X 2018 Expression profile analysis of circular RNAs in ovarian endometriosis by microarray and bioinformatics. Medical Science Monitor 24 9240-9250. (https://doi. org/10.12659/MSM.913885)

Zhang M, Wang S, Tang L, Wang X, Zhang T, Xia X \& Fang X 2019 Downregulated circular RNA hsa_circ_0067301 regulates epithelialmesenchymal transition in endometriosis via the miR-141/Notch signaling pathway. Biochemical and Biophysical Research Communications 514 71-77. (https://doi.org/10.1016/j.bbrc.2019.04.109)

Zhong S, Wang J, Zhang Q, Xu H \& Feng J 2018 CircPrimer: a software for annotating circRNAs and determining the specificity of circRNA primers. BMC Bioinformatics 19 292. (https://doi.org/10.1186/s12859018-2304-1)

Received 11 November 2019

First decision 6 February 2020

Revised manuscript received 27 June 2020

Accepted 6 July 2020 